\title{
Commande avancée pour la régulation des aménagements hydrauliques
}

\author{
Advanced Control for Hydro-Powerplants
}

par A. Libaux

EDF/CNEH Savoie Technolac

et E. Irving

Conseiller Scientifique SUPELEC

This paper presents new solutions for the control design of Hydropower plants. The chosen example is a run of the river type operations. Some control problems in France or in foreign countries have lead to a verification of the existing controls in these plants, and also incited the instigation of advanced controls, carefiully compared to the classical one.

The need for adequate models, as well as for powerful tools and algorithms for control design is a long standing issue. Non-linear partial derivative complicate models, which have been carefully tested through experience and used for classical control design using a trial and error approach, have been in use for a long time. Subsequently, transfer functions were fulfilled by the identification of the complex numerical model time-responses. Indeed, this approach has not been efficient enough, as some dynamic term effects could not be identified due to the nonlinear effects.

The modelization approach using non-linear differential models based on a lumped parameter representation of the physical system is much more powerful. Linearized state space models are deduced from the non-linear ones, which allow the design of convenient classical or modern controls.

It is accepted that classical PI are greatly improved by additional loops assuring positivity, but powerful design techniques are missing for a PID plus a stabilising loop. Hence, an original robust design methodology has been implemented which uses dominant poles and modulus margin placement realised by an optimisation technique. As a generalisation of this idea of additional loops, $L Q G$ control using all measurements available is particularly efficient and simulations on non-linear detailed models are presented.

Finally, due to the non-linear behaviour of the plant and in order to obtain best performances at any flow rate, as at any set-point level, a gain-scheduling adaptive system using switching fixed LQG or switching fixed PI with stabilising loop has been implemented and gives excellent results for the adaptive LQG and acceptable ones for the adaptive $P I$.

Both PI and LQG switching controls have been implemented with very efficient bumpless antisaturation components. Switching criteria have been automated according to the plant flow rate and to the maximum reservoir levels. Due to its general nature, this methodology has not only helped to solve run of the river control problems, but has also been used for speed / power control of turbine units with efficient results.

I $\square$ CONDUITE AU FIL DE L'EAU DES AMÉNAGEMENTS EN CHARGE : EXEMPLE D'ASTON

\subsection{Description de l'installation}

La centrale de haute chute d'ASTON dans les Pyrénées est équipée de 4 groupes PELTON (120 MW). Son circuit hydraulique comprend sur la branche Ariège, le bassin de Luzenac (réservoir 1) entonnant les débits turbinés par la centrale de L'Hospitalet et alimentant la galerie Ariège et sur la branche Aston, le bassin brise-charge recevant l'eau du jet creux de la retenue de Riète (réservoir 2 ) et alimentant la galerie d'Aston. La section moyenne du bassin de Luzenac est de $2000 \mathrm{~m}^{2}$, celle du bassin brise-charge sous Riète n'est que de $100 \mathrm{~m}^{2}$ en partie haute et la section de la cheminée est de $78 \mathrm{~m}^{2}$. Les deux galeries se rejoignent en une galerie 


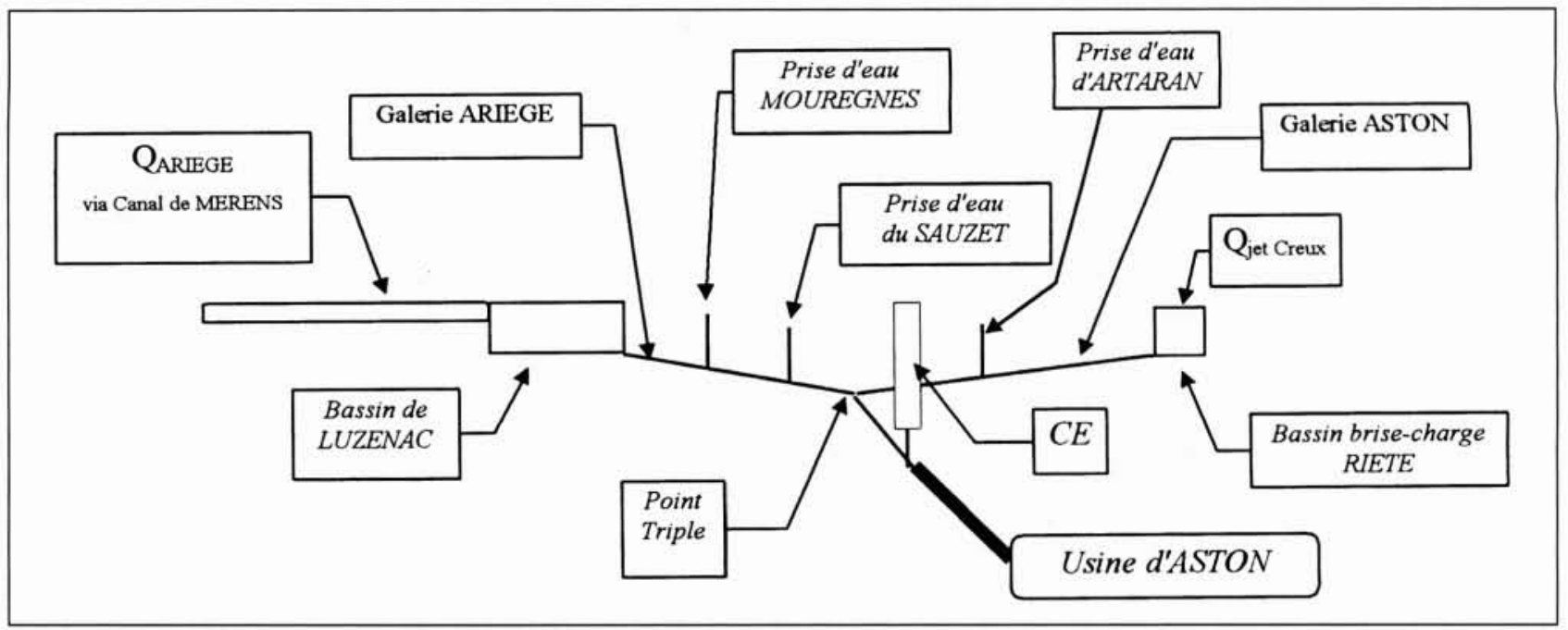

1. Vue d'ensemble de l'Aménagement d'ASTON (GEH Ariège).

commune, puis la cheminée d'équilibre et enfin 2 conduites forcées parallèles dissymétriques alimentant chacune 2 groupes Pelton ( 2 fois $5,5 \mathrm{~m}^{3} / \mathrm{s}$ sur l'une et 2 fois $7,5 \mathrm{~m}^{3} / \mathrm{s}$ sur l'autre soit un débit d'équipement de $26 \mathrm{~m}^{3} / \mathrm{s}$ ).

La régulation doit aider la conduite de l'aménagement par une bonne maîtrise des niveaux des réservoirs ou bassins de mise en charge. L'objectif d'une telle conduite consiste à :

- maximiser la chute sur l'Aménagement (énergie) ;

- éviter les déversements (économie) ;

- maintenir la consigne avec le minimum d'écart en régime stabilisé (précision) ;

- maîtriser les écarts transitoires par rapport au niveau de consigne (stabilité), notamment le dénoyage des galeries d'amenée et les phénomènes de battement de la surface libre susceptibles de détériorer le Génie Civil ;

- éviter d'exciter les dynamiques oscillatoires qui pourraient en retour fatiguer les organes de manœuvre ;

Or, la conduite au fil de l'eau des aménagements de moyenne ou haute chute pose parfois problème: la présence d'une cheminée d'équilibre qui a naturellement tendance à détourner toutes les variations de débit usine, la longueur des galeries, la présence de plusieurs bassins amont, leurs dimensions, le comportement hydraulique dynamique fortement non-linéaire en fonction du débit, sont les causes principales de la difficulté du réglage.

Le cas de l'Usine d'Aston est un bon exemple puisqu'il cumule l'ensemble de ces caractéristiques (également, le projet DILEK GÜROLUK en Turquie, dont EDF/CNEH a réalisé les études d'avant-projet présente la même topologie avec des bassins de plus grande superficie mais en contrepartie une plus grande variabilité du débit, des adductions plus longues et une cheminée de section réduite).

A l'origine de l'automatisation d'Aston et après plusieurs tentatives de réglage sur site d'un régulateur PI sur le niveau du bassin de Luzenac, il fut finalement décidé d'implanter un asservissement des groupes au seul niveau cheminée et les capteurs de niveau aux bassins amont furent supprimés. Ce faisant, il s'est avéré impératif de temporiser fortement les actions de commande de façon à ne provoquer aucune oscillation du niveau cheminée. Outre la lenteur de cette régulation, l'absence d'action intégrale ne permet pas d'éviter de longues plages de déversement aux bassins, trompeuses car propices à une stabilisation radicale des niveaux.

Lorsqu'on étudie une loi de commande pour de tels aménagements, la démarche classique est de paramétrer le régulateur principal de type PI/PID sur le niveau du bassin à régler, puis de lui adjoindre des boucles correctrices, comme par exemple, une boucle auxiliaire à action dérivée filtrée sur le niveau cheminée $[9,11,12]$ en plus de la boucle principale ou bien, l'utilisation d'une combinaison linéaire de plusieurs niveaux. Si un paramétrage "satisfaisant » peut être proposé, la synthèse d'une loi de commande «optimale » en tout point de fonctionnement demeure un objectif difficile.

\subsection{Modélisation par représentation d'état}

Pour concevoir un régulateur adapté au problème, un modèle mathématique à base d'équations différentielles non-linéaires sous forme d'état a été développé à partir des équations nonlinéaires du coup de bélier de masse dans les galeries, du coup de bélier d'onde dans les conduites forcées, et des équations régissant les variations de niveau-débit dans la cheminée d'équilibre et les bassins de mise en charge. La linéarisation de ce modèle a fourni un modèle linéaire d'état de même ordre, ce qui a permis de déterminer les différentes fonctions de transfert [12]. Cette démarche a permis de détecter des écarts importants, concernant les caractéristiques fréquentielles, avec la méthode classique d'identification faite à partir de réponses temporelles issues du logiciel BELIER (modes oscillatoires haute ou très basse fréquence incorrectement représentés).

Le système hydraulique est un système dynamique soumis à des entrées (composantes du vecteur $u(t))$ et fournissant des sorties (composantes du vecteur $y(t)$ ) délivrant des informations dont le comportement est entièrement caractérisé par un vecteur d'état $x(t)$ de dimension $n$ lié aux entrées et aux sorties par les relations suivantes:

$$
\left\{\begin{array}{l}
\frac{d x(t)}{d t}=f(x(t), u(t)) \\
y(t)=h(x(t), u(t))
\end{array}\right.
$$

Ces deux équations - équation d'évolution et équation de la mesure - constituent la représentation d'état du système dynamique. Dans notre cas, le vecteur d'entrée $u(t)$ prend la forme :

$$
u(t)=\left[Q_{U S}(t) Q_{e 1}(t) Q_{e 2}(t)\right]^{T}
$$

$Q_{U S}(t)$ est le débit usine, variable de commande, égal au débit dans la conduite forcée équivalente, $Q_{e l}(t)$ et $Q_{e 2}(t)$ sont les débits entrants dans les deux bassins 1 et 2 qui sont 
des variables d'entrée perturbatrices du vecteur $x(t)$ d'état donné par:

$$
x(t)=\left[Z_{1}(t) Z_{2}(t) Z_{C E}(t) Q_{G 1}(t) Q_{G 2}(t) Q_{T C}(t) H_{P T}(t) Q_{C F}(t) H_{U S}(t)\right]^{T}
$$

où $Z_{1}(t), Z_{2}(t)$ et $Z_{C E}(t)$ sont respectivement les niveaux des réservoirs 1,2 et de la cheminée d'équilibre, $Q_{G I}(t), Q_{G 2}(t)$, $Q_{T C}(t), Q_{C F}(t)$ sont les débits dans les galeries d'amenée, la galerie commune et la conduite forcée, $H_{P T}(t), H_{U S}(t)$ sont les pressions totales au point triple et dans l'usine. Les niveaux étant mesurés, le vecteur d'observation $y(t)$ est donné par:

$$
y(t)=\left[Z_{1}(t) Z_{2}(t) Z_{C E}(t)\right]^{T}
$$

Selon la formulation choisie, l'équation d'état ( 1 et 2), dans sa forme la plus générale, est issue des trois équations suivantes :

$$
\left\{\begin{aligned}
\frac{d x(t)}{d t} & =\phi(x(t), u(t), z(t)) \\
0 & =g(x(t), u(t), z(t)) \\
y(t) & =\varphi(x(t), u(t), z(t))
\end{aligned}\right.
$$

$z(t)$ peut être explicité par :

$$
z(t)=\psi(x(t), u(t))
$$

ce qui permet de résoudre explicitement le système (6) et (7) par:

$$
\mathrm{d} x(t) / \mathrm{d} t=\phi(x(t), \psi(x(t), u(t)), u(t))
$$

par exemple, à l'aide du schéma d'Euler :

$$
x\left(t+T_{e}\right)=x(t)+T_{e} \cdot \phi(x(t), \psi(x(t), u(t)), u(t))
$$

$T_{e}$ étant l'intervalle d'échantillonnage.

' $\mathrm{La}$ linéarisation de la représentation d'état est alors développée autour d'un régime d'équilibre :

$$
\left\{\begin{array}{l}
\Delta \dot{x}(t)=A \Delta x(t)+B \Delta u(t) \\
\Delta y(t)=C \Delta x(t)+D \Delta u(t)
\end{array}\right.
$$

dans laquelle on appelle $A$ la matrice d'évolution de dimension $n \times n, B$ la matrice de commande de dimension $n \times m$, $C$ la matrice d'observation de dimension $l \times n$ et $D$ la matrice de transmission directe $l \times m$.

$\Delta u(t), \Delta y(t)$ et $\Delta x(t)$ sont respectivement les écarts définis par :

$$
\begin{aligned}
& \Delta u(t)=u(t)-u_{0} \\
& \Delta x(t)=x(t)-x_{0} \\
& \Delta y(t)=y(t)-y_{0}
\end{aligned}
$$

des variables $u(t), y(t)$ et $x(t)$ par rapport à leurs valeurs d'équilibre $u_{0}, y_{0}$ et $x_{0}$.

La représentation d'état non-linéaire a donné le schéma MATLAB-SIMULINK représenté (fig. 2) dans lequel on retrouve la structure topologique de l'aménagement.

Cette modélisation a fait l'objet d'une validation code à code avec le logiciel BELIER 5.0 d'EDF lui-même largement validé comparativement à des mesures sur site.



2. Schéma SIMULINK de l'Aménagement d'Aston. 
1.3 Régulateur linéaire quadratique gaussien (LQG)

Le régulateur LQG [1] est composé de 2 éléments :

- un filtre de Kalman permettant d'estimer l'état du modèle ;

- un régulateur d'état linéaire quadratique utilisant cet état estimé.

Le filtre de Kalman ou observateur d'état est un modèle, recalé par sa sortie, du système à régler qui satisfait aux relations :

$$
\begin{array}{r}
d x_{e}(t) / d t=A_{e} x_{e}(t)+B_{e 1} u(t)+B_{e 2} Q_{e 1 e}(t)+B_{e 3} Q_{e 2 e}(t) \\
\quad+K_{x}\left[y(t) \pm y_{e}(t)\right] \\
d Q_{e 1 e}(t) / d t=K_{Q e 1}\left[y(t) \pm y_{e}(t)\right] \\
d Q_{e 2 e}(t) / d t=K_{Q e 2}\left[y(t) \pm y_{e}(t)\right] \\
d e(t) / d t=y_{r}(t) \pm r(t)
\end{array}
$$

$y_{r}(t)$ est la variable à régler et $r(t)$ sa consigne.

Le régulateur d'état à critère quadratique fournit une commande $u(t)$ qui minimise le critère $J$ donné par:

$$
J=\lim _{T \rightarrow \infty} E\left\{1 / T \int_{0}^{T} x_{a}(t)^{T} Q_{a} x_{a}(t)+u_{l}(t)^{T} R_{l} u_{l}(t) d t\right\}
$$

où $x_{a}(t)$ est l'état augmenté comprenant l'état du système à régler augmenté de $e(t), Q_{e l}(t)$ et $Q_{e 2}(t), u_{I}(t)=\mathrm{d} u(t) / \mathrm{d} t$ appelée commande interne.

Ce régulateur d'état satisfait à :

$$
\begin{aligned}
& u(t)= \pm G_{x} x_{e}(t) \pm G_{Q e 1} Q_{e l e}(t) \pm G_{Q e 2} Q_{e 2 e}(t) \pm G_{e} e(t) \\
& y_{e}(t)=\left[\begin{array}{l}
Z_{1 e}(t) \\
Z_{2 e}(t) \\
Z_{C e}(t)
\end{array}\right]=C x_{e}(t) \quad(7), \quad y(t)=\left[\begin{array}{l}
Z_{1}(t) \\
Z_{2}(t) \\
Z_{C}(t)
\end{array}\right]
\end{aligned}
$$

Les termes $K_{x}, K_{Q_{e l}}, K_{Q_{e} 2}, G_{x}, G_{Q_{e} 1}, G_{Q_{e} 2}$ et $G_{e}$ sont obtenus en résolvant deux équations de Riccati dont les coefficients sont des fonctions des matrices $A, B, C, D, Q_{a}, R_{l}$ et des matrices de covariances des bruits de mesure.

Les coefficients de pondérations $Q_{a}$ et $R_{l}$ peuvent le cas échéant être ajustés et sont a priori déterminés en pénalisant les états dont l'amplitude de variation est jugée trop importante.

Un avantage fort de cette méthode est - par le principe de l'observateur unique - de pouvoir traiter globalement plusieurs mesures ce qui, dans notre cas, est nécessaire. Or une commande utilisant à la fois toutes les mesures de niveau disponibles est une généralisation dynamique du régulateur à plusieurs boucles de rétroaction dont on a vu précédemment qu'il s'imposait au cas présent. La raison théorique $[3,4]$ de cette amélioration peut être trouvée dans le fait que les zéros du système en boucle ouverte peuvent être déplacés par l'adjonction de plusieurs boucles de rétroaction. Or, des zéros stables à dynamique haute fréquence fournissent de bonnes performances.

Deux étapes doivent être franchies : d'abord, déterminer les coefficients de pondération pour fournir des réponses satisfaisantes pour un point de fonctionnement bien choisi, ensuite, assurer la robustesse du régulateur ainsi conçu pour les autres points de fonctionnement. Cette dernière condition est satisfaite si l'on choisit un point de conception correspondant au modèle linéarisé le moins stable. Comme il sera nécessaire d'utiliser plusieurs régulateurs LQG commutant entre eux pour régler, soit le niveau d'un réservoir, soit l'autre, une technique d'antisaturation adaptée au régulateur LQG a été mise au point. Elle permet, en outre, de gérer de manière efficace les saturations de la commande.

Devant la non-linéarité du problème et afin de conserver de bonnes performances à tous débits, il s'est avéré nécessaire de concevoir un type original de régulation adaptative. Deux régulateurs LQG, l'un pour les débits élevés, l'autre pour les faibles débits, ont été conçus et l'adaptation des régulateurs a été réalisée par une logique de commutation fonction du débit usine. Pour éliminer les à-coups sur la commande au moment de la commutation des régulateurs, une anti-saturation a été introduite dans la conception LQG.

En fait, c'est l'équivalent de 4 régulateurs LQG qui est utilisé. Chacun des LQG ayant 2 sorties commande, l'une pour le bassin $n^{\circ} 1$ et l'autre pour le $n^{\circ} 2$. La commutation

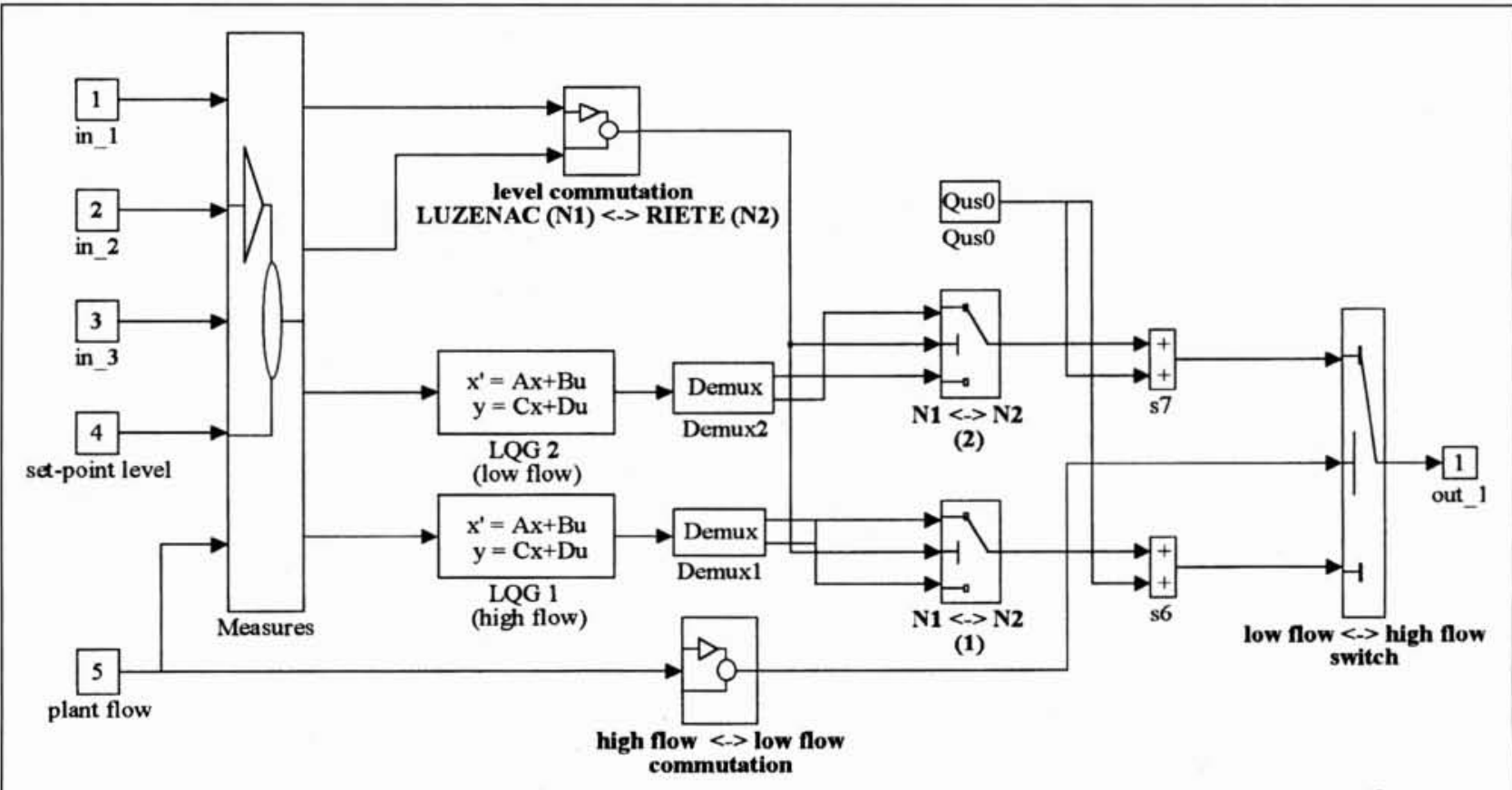

3. Régulateur auto-adaptatif (double LQG avec logique de commutation en fonction du débit et logique de commutation sur le niveau bassin à régler). 




4. Réponse temporelle du double régulateur LQG sur scénario complexe et visualisation de la logique de commutation niveau et débit.

sur la commande d'un bassin donné est réalisée quand son niveau devient le plus élevé (danger de déversement).

La simulation présentée (fig. 4) montre la performance d'ensemble en des points de fonctionnement très variés puisque comme le montre le graphique du bas, les débits entrants amonts suivent d'importantes variations et ce dans un environnement extrêmement bruité (écart-type du bruit blanc égal à $1 \mathrm{~cm}$ ). Le graphique intermédiaire présente les variables logiques de commutation d'un régulateur sur l'autre (haut et bas débit) et d'un niveau bassin sur l'autre. Le graphe supérieur montre que les niveaux, lorsqu'ils font l'objet du réglage à la consigne, sont particulièrement bien tenus. Les oscillations de niveau dans la cheminée sont bien contrôlées. La consigne de niveau a été volontairement choisie haute $(70 \mathrm{~cm}$ sous la cote de déversement) et le pourcentage de volume perdu par déversement par rapport au volume total turbiné pendant cette séquence est inférieur à $0,6 \%$.

\section{II —ANALYSE DU LQG ET D'UN RÉGU- LATEUR PI AVEC BOUCLE STABILI- SATRICE NIVEAU CHEMINÉE}

\subsection{Propriétés fréquentielles du régulateur LQG à bas débit}

Le système à régler présente un comportement plus oscillatoire à faible débit par diminution des pertes par frottement dans les conduites. Dans ce qui suit, nous exposons la démarche suivie partant de l'analyse fréquentielle comparée de deux régulateurs jusqu'à la synthèse du régulateur nonlinéaire PI.

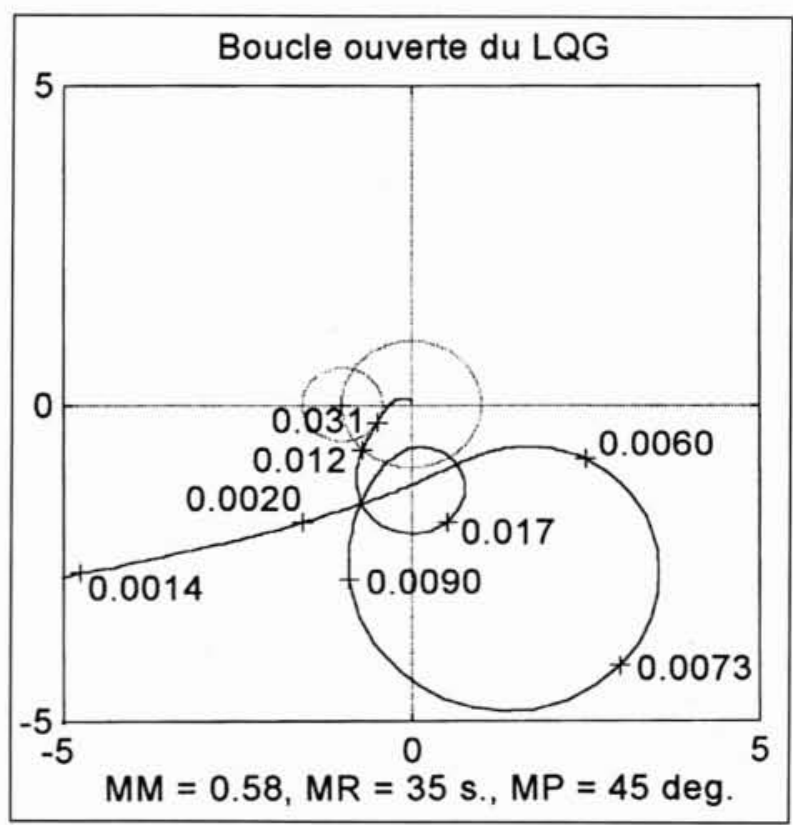

5. Diagramme de Nyquist de la boucle ouverte du régulateur LQG conçu à $5 \mathrm{~m}^{3} / \mathrm{s}$ et pour le bassin de Riète (les pulsations indiquées par des + sur la boucle ouverte sont en $\mathrm{rad} / \mathrm{s}$ ).

La fonction de transfert en boucle ouverte du LQG présentée (fig. 5) appelle plusieurs remarques :

- les basses fréquences correspondent bien à un déphasage de -180 degrés représentant les déphasages basses fré- 


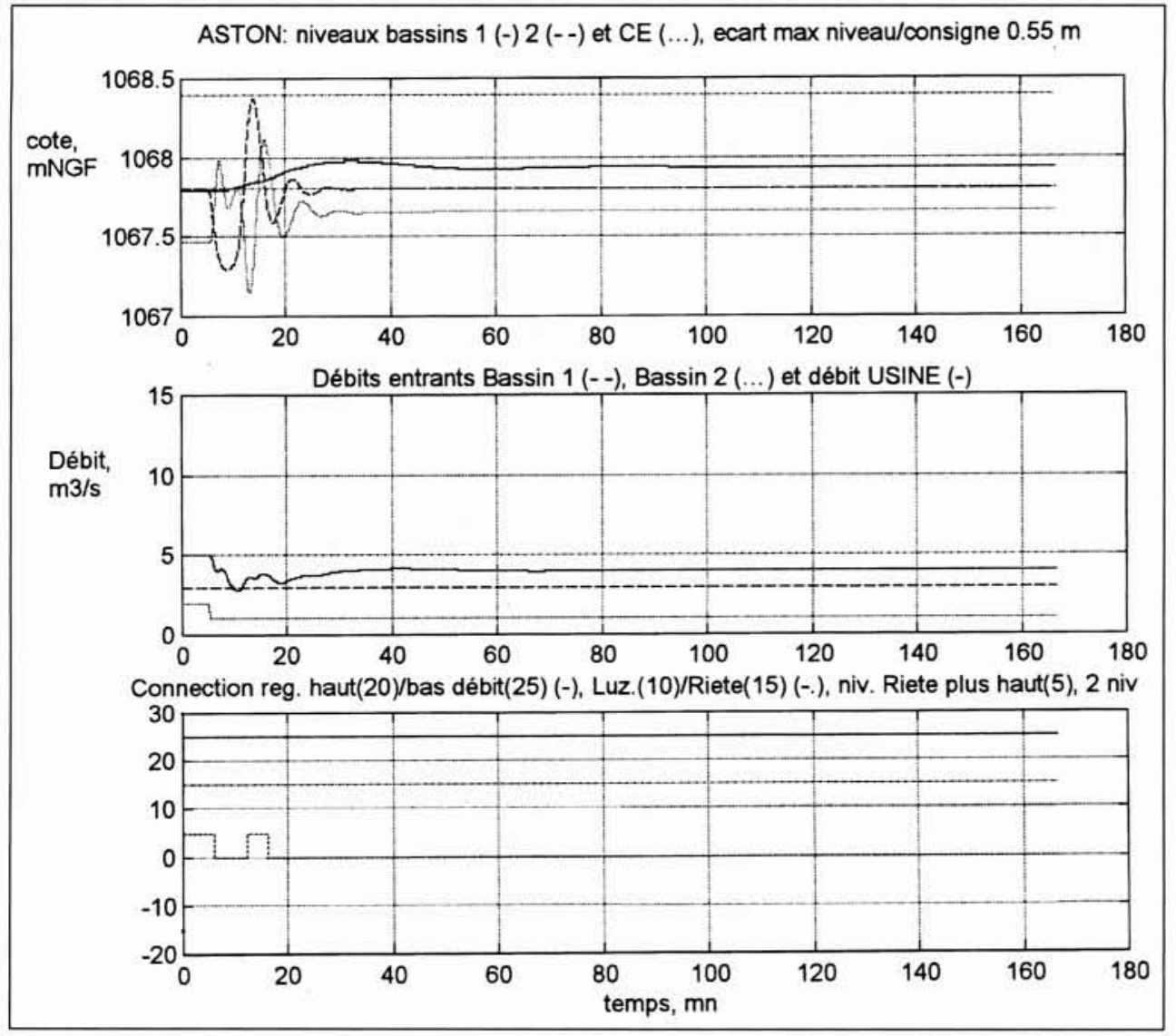

6. Réponses du LQG à $5 \mathrm{~m}^{3} / \mathrm{s}$ pour le régulateur conçu à ce même débit sur le bassin $n^{\circ} 2$ : ce qui est remarquable est le temps de réponse inférieur à 20 minutes pour le bassin $n^{\circ} 2$ (simulation avec modèle complet non-linéaire).

quences de l'intégrateur du système à régler et de l'intégrateur du régulateur $\mathrm{LQG}$;

- la marge de module $M M=0,58$ est acceptable, le minimum de la distance au point critique $[-1,0]$ est obtenu à la pulsation de $0,032 \mathrm{radian} / \mathrm{s}$, soit une période de $196 \mathrm{~s}$, ce qui assure des performances correctes ;

- la marge de retard $M R=35 \mathrm{~s}$ montre que les filtres antirepliement et capteurs de constantes de temps 30 et $10 \mathrm{~s}$ doivent être intégrés au modèle de conception ;

- le gain de 5 pour une pulsation basse fréquence de 0,0014 radian/s, soit une constante de temps de $710 \mathrm{~s}$, montre que la ralliement à la consigne va s'opérer dans des durées de l'ordre de $20 \mathrm{mn}$, ce qui satisfait aux spécifications ;

- ces bonnes performances s'expliquent par le fait que le lobe à la pulsation 0,00733 , soit une période de $860 \mathrm{~s}$, a sa phase correctement avancée par rapport au mode bassin amont. Cela n'était pas le cas du régulateur PI, même avec sa boucle additionnelle qui était efficace seulement pour le mode cheminée de pulsation $0,01727 \mathrm{radian} / \mathrm{s}$ de période $363 \mathrm{~s}$.

Le régulateur ainsi conçu donne une stabilisation des $3 \mathrm{ni}$ veaux dans des temps de réponse inférieurs à $20 \mathrm{mn}$ (fig.6).

De manière similaire aux régulateurs LQG, c'est un ensemble de 4 régulateurs PI (avec boucle stabilisatrice) commutant entre eux qui est utilisé pour obtenir des performances acceptables aussi bien à haut qu'à bas débit pour le réglage de niveau du bassin $n^{\circ} 1$ ou du bassin $n^{\circ} 2$.

\subsection{Méthodologie de synthèse du régulateur PI avec filtre cheminée}

Il a été constaté que l'usage d'un simple régulateur PI était très limitateur du point de vue des performances. Le diagramme de Nyquist de la boucle ouverte du PI avec le système à régler vu des niveaux bassins permet d'expliquer ces limitations.
En effet, sur la boucle ouverte du PI de coefficients $K_{P I}=$ 0,5 et $T_{P I}=2500 \mathrm{~s}$, plusieurs remarques peuvent être faites : - les termes basse fréquence ont un déphasage de -180 degrés qui correspond à l'addition de 2 fois -90 degrés des intégrateurs du système à régler et du régulateur PI ;

- l'oscillation qui correspond à la pulsation de 0,0076 radians/s a une période de $822 \mathrm{~s}$ qui représente l'oscillation bassin. Le

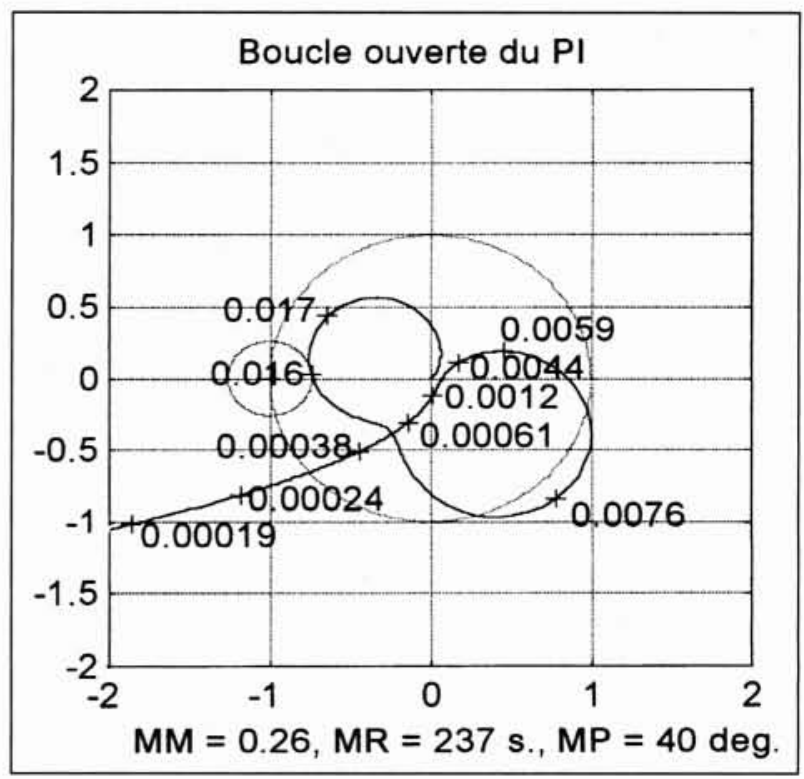

7. Diagramme de Nyquist de la boucle ouverture du régulateur PI conçu à $5 \mathrm{~m}^{3} / \mathrm{s}$ et pour le bassin de Riète (les pulsations indiquées par des + sur la boucle ouverte sont en $\mathrm{rad} / \mathrm{s}$ ). 
lobe est bien placé pour l'amélioration des performances par augmentation du gain, parce qu'il est principalement dans le demi-plan positif opposé au point critique $(-1,0)$;

- ce qui est limitateur, du point de vue des performances, est le lobe en haut à gauche qui a comme pulsation 0,0173 radians/s de période de $362 \mathrm{~s}$ qui correspond à l'oscillation cheminée. En effet, si on augmente le gain du régulateur, la marge de module qui est déjà de 0,2615 , c'est-à-dire trop faible, va être encore diminuée, ce qui est inacceptable.

Or ces valeurs du PI seul donnent des performances tout à fait insuffisantes : quelques heures de temps de réponse avec un gain donné ci-dessus trop grand pour satisfaire à une marge de robustesse acceptable.

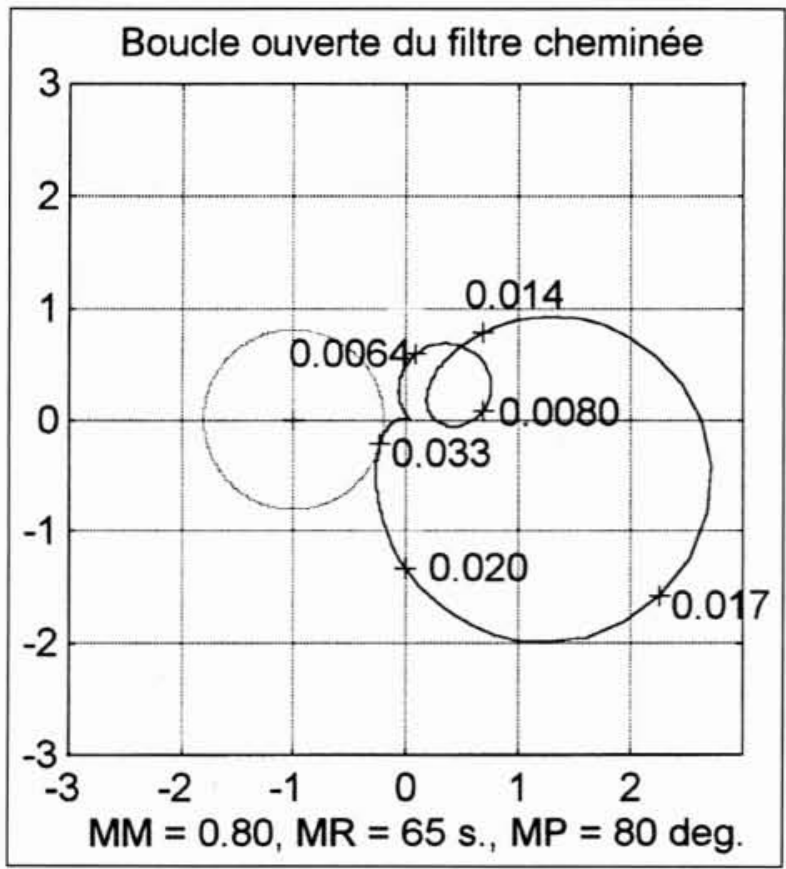

8. Diagramme de Nyquist de la boucle ouverte de l'action dérivée filtrée " cheminée " à $5 \mathrm{~m}^{3} / \mathrm{s}$ et pour le bassin de Riète (les pulsations indiquées par des + sur la boucle ouverte sont en $\mathrm{rad} / \mathrm{s}$ ).

Examinons le diagramme de Nyquist de la boucle ouverte du filtre cheminée de fonction de transfert $K_{C H} p /\left(p+1 / T_{C H}\right)$ avec $K_{C H}=1, T_{C H}=100$ (fig. 8 ). Ce diagramme indique que le lobe le plus grand qui correspond à la pulsation 0,017 . c'est-à-dire à la période $354 \mathrm{~s}$ de l'oscillation cheminée est opposée au lobe cheminée correspondant dû à la boucle ouverte par le régulateur PI. Il sera donc possible d'augmenter grandement le gain du PI, même en faisant franchir à sa boucle ouverte le point critique $(-1,0)$, et de compenser le gonflement correspondant vers le haut à gauche du PI par un gonflement exactement opposé vers le bas à droite dû au filtre cheminée. Il est ainsi possible d'évaluer la relation qui doit lier $K_{P I}$ à $K_{C H}$ pour que les diamètres des lobes cheminée soient sensiblement égaux. Cette relation est $K_{P l} / K_{C H} \cong$ 156. Ainsi, il est possible d'arriver à un gain du PI qui soit suffisamment grand pour obtenir des performances acceptables au petit débit de $5 \mathrm{~m}^{3} / \mathrm{s}$ pour le cas difficile du bassin $\mathrm{n}^{\circ} 2$ en cote haute (avec sa section de l'ordre de $100 \mathrm{~m}^{2}$ alors que le bassin $\mathrm{n}^{\circ} 1$ peut avoir $2500 \mathrm{~m}^{2}$ ).

Après une procédure d'optimisation par placement des pôles et marge de robustesse (marge de module), qui sera détaillée plus loin, les coefficients suivants sont obtenus : $K_{P I}=3,38, \quad T_{P I}=775,8 \mathrm{~s} \quad K_{C H}=2,31 \quad T_{C H}=2943,73 \mathrm{~s}$ Ils fournissent le diagramme de Nyquist donné (fig. 9) et les pôles de la boucle fermée sur les figures suivantes. Le pre- mier point obtenu avant la procédure d'optimisation marqué de « $+»$ avait une marge de module insuffisante de 0,43 . La procédure d'optimisation a permis d'obtenir 0,5 en améliorant l'amortissement (points marqués « $x »)$, tout en ralentissant fort peu les modes lents.

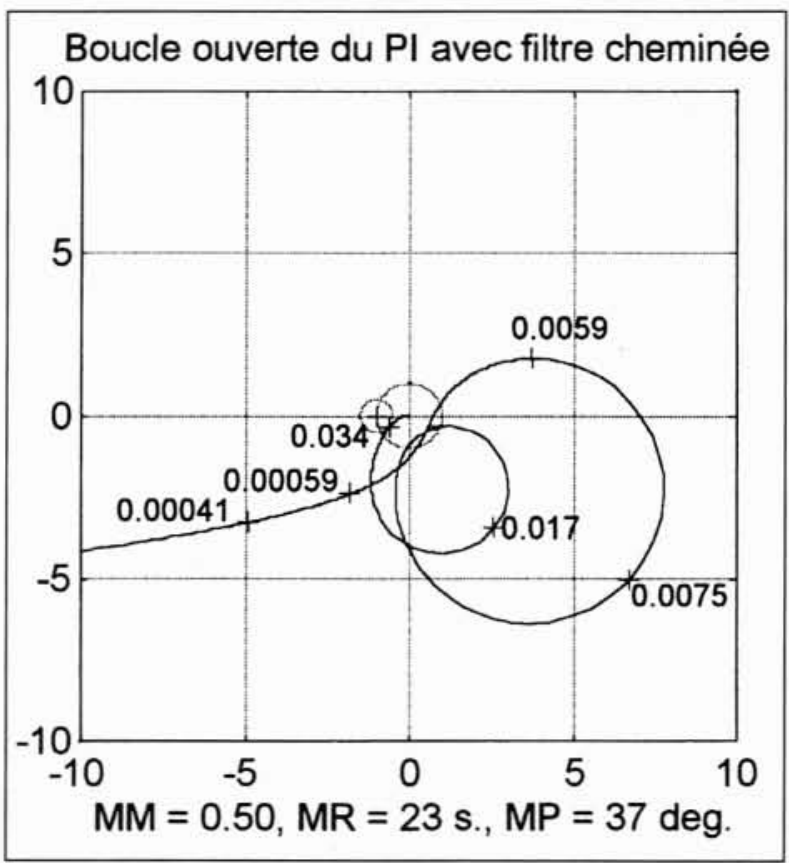

9. Diagramme de Nyquist du régulateur PI avec action dérivée filtrée " cheminée " $\left(K_{P I}=\mathbf{3 , 4}, T_{P I}=776 \mathrm{~s}\right.$, $K_{C H}=2,3, T_{C H}=2940 \mathrm{~s}$ ) trouvés par placement des pôles et de la marge de module.

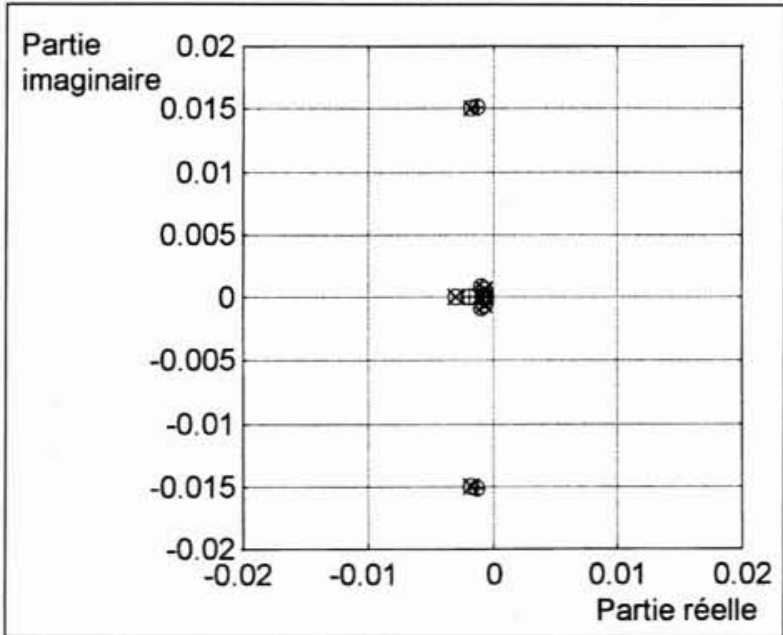

10. Pôles du régulateur PI avec action dérivée filtrée " cheminée " $\left(K_{P I}=3,4, T_{P I}=776 \mathrm{~s}, K_{C H}=2,3\right.$, $\left.T_{C H}=2940 \mathrm{~s}\right)$.

Les différents graphiques précédents ont une grande importance parce qu'ils montrent deux enseignements importants :

1) Une méthodologie efficace de détermination du PI avec filtre cheminée a été mise au point. Elle généralise les approches de conception robuste telles que QFT [5] au cas des systèmes à plusieurs boucles en introduisant des critères de placement des pôles simultanément à ceux de robustesse. Contrairement aux méthodes classiques de placement des 




11. Réponses du régulateur PI avec filtre cheminée à $5 \mathrm{~m}^{3} / \mathrm{s}$ pour le régulateur conçu à ce même débit sur le bassin $\mathrm{n}^{\circ} 2$ $\left(K_{P I}=3,4, T_{P I}=776 \mathrm{~s}, K_{C H}=2940 \mathrm{~s}\right)$.

pôles, qui nécessitent un régulateur ayant un grand nombre de degrés de liberté (tel que le RST d'Aström-Wittenmark [2]), la méthodologie utilisée permet de régler les paramètres d'un régulateur ayant aussi peu de degrés de liberté que le régulateur PI avec filtre cheminée de l'étude. Bien entendu, ce faible nombre de degrés de liberté génère des limitations de performances qui sont mises très clairement en évidence par l'approche.

2) La supériorité de l'approche LQG est manifeste. C'est, à la fois l'ordre élevé du régulateur, qui est proche de l'ordre du système à régler et la méthode de détermination de ses degrés de liberté qui donnent de bons résultats. La diminution des temps de réponse et l'augmentation de l'amortissement sont sensiblement dans un rapport de l'ordre de 3 . Il faut souligner que ces bons résultats se vérifient sur les simulations présentées qui utilisent un modèle non linéaire complet du système à régler.

La méthode pour trouver les variations des paramètres qui fournissent un déplacement des pôles dans un sens voulu avec une marge de module donnée se divise en plusieurs étapes :

- les pôles les plus critiques sont d'abord repérés ;

- le sens de variation des paramètres est trouvé en choisissant le sens favorable de déplacement des pôles sur le groupe critique ;

- l'effet de ces sens de variation sur les autres groupes de pôles est examiné ;

- si ce sens de variation est favorable, alors la variation du paramètre peut être augmentée ;

- si ce sens est défavorable, un compromis doit être adopté qui fige la variation du paramètre ;

- une variation quantitative des paramètres est obtenue en ajoutant au critère de déplacement des pôles, qui permet d'améliorer l'amortissement et/ou de diminuer la lenteur des modes les plus lents, des considérations de robustesse, par exemple, la satisfaction d'une marge de module désirée $M M_{D}$ (choisie égale à 0,5 , ce qui permet d'obtenir une marge de gain $\geqslant 2$ ). Décrivons, plus précisément cette dernière approche.

\subsection{Détermination du PI et filtre cheminée par place- ment des pôles les plus critiques avec satisfaction d'une marge de module désirée}

Une fois obtenus la structure et l'ordre de grandeur des coefficients du PI avec filtre cheminée par des considérations qualitatives du type fréquentiel comme décrit précédemment, il est intéressant de disposer d'une méthode quantitative qui permette de déplacer directement les pôles les plus critiques tout en satisfaisant une marge de robustesse adéquate. En utilisant l'approche décrite dans la thèse d'Yves Piguet (EPFL Lausanne 1997) qui consiste à plonger le problème qui vient d'être décrit dans une méthode générale d'optimisation pour fonctions non définies explicitement, on aboutit à la formulation suivante incluant à la fois des critères de placement des pôles et de robustesse.

Dans l'ensemble des pôles de la boucle fermée, on repère un nombre de pôles critiques égal à la moitié du nombre de degrés de liberté du régulateur PI avec filtre cheminée qui est de $4\left(K_{P I}, T_{P I}, K_{C H}\right.$, et $\left.T_{C H}\right)$, soit une paire de pôles. Dénommons par:

- $\mu_{C H}=\mu_{C H R}+i \mu_{C H C}$ le mode cheminée ;

- $\mu_{P I}=\mu_{P I R}+i \mu_{P I C}$ le mode le plus lent de l'intégrateur ;

- $M M$ la marge de module (minimum de la distance de la boucle ouverte au point critique $(-1,0))$;

Les 3 quantités correspondant au jeu de paramètre $K_{P I}$, $T_{P l}, K_{C H}$ et $T_{C H}$.

Le problème de synthèse des paramètres $K_{P l}, T_{P l}, K_{C H}$ et $T_{C H}$ consiste à trouver les variations de ces paramètres qui minimisent le critère $J\left(K_{P l}, T_{P l}, K_{C H}, T_{C H}\right)$ défini par :

$$
\begin{aligned}
J\left(K_{P l}, T_{P l}, K_{C H}, T_{C H}\right)= & \lambda_{C H}^{2}\left|\mu_{C H D} \pm\left(\mu_{C H}\left(K_{P l}, T_{P l}, K_{C H}, T_{C H}\right)\right)\right|^{2} \\
& +\lambda_{P l}^{2}\left|\mu_{P I D} \pm\left(\mu_{P l}\left(K_{P l}, T_{P l}, K_{C H}, T_{C H}\right)\right)\right|^{2} \\
& +\lambda_{M M}^{2}\left(M M_{D} \pm M M\left(K_{P l}, T_{P l}, K_{C H}, T_{C H}\right)\right)^{2}
\end{aligned}
$$


$\mu_{C H D}, \mu_{P I D}$ et $M M_{D}$ étant les valeurs désirées des modes critiques et de la marge de module. Les coefficients de pondération $\lambda_{C H}, \lambda_{P I}$, et $\lambda_{M M}$ sont choisis de façon à marquer l'importance attachée aux différents termes.

La résolution du problème précédent peut être effectuée à l'aide d'un programme général d'optimisation qui ne nécessite pas la connaissance explicite des gradients des différentes fonctions $\mu_{C H}, \mu_{P I}$ et $M M$ par rapport à $K_{P I}, T_{P l}, K_{C H}$, et $T_{C H}$.

Mais avant de mettre en œuvre cette procédure qui peut conduire à des temps calcul prohibitifs et à des blocages des itérations qu'il est malaisé d'analyser, il est intéressant de vérifier qu'une méthode simple fonctionne convenablement. Cette méthode simple consiste à optimiser le critère précédent par une méthode de Newton-Gauss utilisant des gradients déterminés numériquement (méthode de Marquardt). On doit ainsi effectuer les opérations successives suivantes :

1) Calcul numérique des gradients $g_{C H}, g_{P I}$ et $g_{M M}$ des fonctions $\mu_{C H}$, $\mu_{P l}$ et $M M$ par rapport à $K_{P l}, T_{P I}, K_{C H}$ et $T_{C H}$;

2) Estimation par méthode de Newton-Gauss des variations des paramètres du régulateur :

3) Choix des différents paramètres. Le comportement des pôles $f$ par rapport au vecteur des paramètres du régulateur est fortement non-linéaire, la fonction $M M$ étant beaucoup plus linéaire particulièrement par rapport à $K_{P I}$ et $K_{C H}$. Les valeurs propres désirées $\mu_{C H D}$ et $\mu_{P I D}$ doivent être choisies peu différentes des valeurs propres initiales $\mu_{C H O}$ et $\mu_{P l 0}$, par contre, vu la linéarité de la fonction, $M M, M M_{D}$ peut être imposée immédiatement à la valeur souhaitée, par exemple 0,5 .

4) Résultats numériques: les figures précédentes montrent que la méthodologie proposée est efficace, à condition de choisir une progression modérée des valeurs propres. D'autre part, la nature limitatrice de la structure du régulateur PI avec filtre cheminée se constate aisément par le fait qu'une augmentation de l'amortissement du mode cheminée entraîne nécessairement un ralentissement des modes intégrateurs. Il semble que, par suite de l'existence du mode bassin, l'amortissement du mode cheminée est limité. On pourra constater que ce n'est pas le cas du régulateur LQG où le mode cheminée est bien amorti en 20 minutes.

5) Généralisations de l'approche : cette méthodologie peut aisément se généraliser à beaucoup plus de fonctions, par exemple, la marge de retard et des critères d'excitation de la commande. Il est possible de rajouter des critères temporels tels que dépassement et temps de réponse etc. Dans ces généralisations, il y a intérêt à conserver des critères fréquentiels de robustesse tels que la marge de module et la marge de retard. Enfin, cette méthode de détermination se généralise au cas multi-modèle qui est celui de l'étude de régulation hydraulique. Dans le cas multi-modèle, ce qui doit être repéré comme pire cas, c'est, en plus du jeu des pires valeurs propres, le jeu des pires modèles.

\section{III —CONCLUSION}

Afin d'être en mesure de résoudre les problèmes rencontrés en exploitation hydraulique, la méthodologie d'étude des régulations a été complètement repensée. Cette méthodologie s'appuie sur un ensemble logiciel d'aide à la conception de régulateurs particulièrement puissant et complet dans l'environnement MATLAB/SIMULINK. Les méthodes de "Commande Avancée » qui, il faut le reconnaître, tardent à pénétrer la pratique industrielle en particulier hydraulique, mettent à notre disposition une grande variété d'algorithmes tant pour la modélisation que pour la régulation.

Différentes techniques de commande robuste ou adaptative utilisant la représentation d'état non-linéaire et linéarisée du système à régler ont été développées et appliquées à un système hydraulique complexe difficile à régler. Pour cet aménagement, une première méthode de synthèse combinant des régulateurs de type PID adaptatif par commutation suivant le débit d'usine et le type de bassin, chacun à conception origi- nale combinée robuste par placement de pôles à critère fréquentiel, a été développée. Cette première approche a été comparée à une deuxième méthode de commande adaptative également originale combinant des régulateurs LQG antisaturés. L'apport des régulateurs de type LQG est clair par rapport au PID avec boucle stabilisatrice: le nombre de paramètres libres du LQG et sa méthode de conception permettent de satisfaire des spécifications de temps de réponse et d'amortissement impossibles à obtenir avec le PID malgré une méthode de conception robuste particulièrement efficace. Les performances de la commande adaptative tout en étant meilleures que celles utilisant le PID avec boucle stabilisatrice, n'empêchent pas que ces dernières restent très acceptables.

Dans l'avenir, on réservera donc la commande adaptative LQG à des cas d'exploitation particulièrement difficiles et la commande adaptative ou non basée sur des PIB robustes à boucle stabilisatrice ou non pour les cas d'exploitation les plus courants. Cette méthodologie étant générale a également pu être appliquée à la résolution des problèmes de régulation de fréquence et de vitesse des turbines hydrauliques. Elle peut aussi être étendue à la résolution des problèmes de réglage de tension et de puissance réactive sur le réseau électrique de puissance.

\section{RÉFÉRENCES}

[1] Anderson B.D.O., Moore J.B. (1989). - « Optimal Control Linear Quadratic Methods ». Prentice Hall.

[2] Astrom K.J., Wittenmark B. (1990) - «Computer Controlled Systems ». Prentice Hall.

[3] Freudenberg J.S., Looze D.P. (1985). - « Right Half Plane Poles and Zeros and Design Tradeoffs in Feedback Systems ». IEEE Trans. Aut. Control, Vol. AC-30, n 6, pp. 555-565.

[4] Freudenberg J.S., Looze D.P. (1988). - « Frequency Domain Properties of Scalar and Multivariable Feedback System ». Springer-Verlag.

[5] HorowITz I.M. (1993). - «Quantitative Feedback Design Theory (QFT) », Vol. 1, QFT Publications, 4470 Grinnel Ave., Boulder, Colorado 80303.

[6] IRving E. (1998). - « Commande Robuste Adaptative». Cours Supélec

[7] Kallath T. (1984). - « Linear System Theory ». Prentice Hall.

[8] Libaux A. (1996). - « Principes de Régulation pour une Conduite Optimale de I'Usine d'Aston ». EDF/CNEH EH DT ED/96 051 A.

[9] Lejeune A., Marques A. (1987). - « Stabilité Hydraulique des Chambres d'Equilibre dans des Systèmes d'Aménagements Complexes ». Thèse de Doctorat, Liège.

[10] File G., Staubl T. (1996). - « Understanding the Dynamic Behaviour of a Hydro Power Plant Using Multiple Input/Output Model-Analysis». Modelling Testing and Monitoring II, Lausanne.

[11] Lein G., Hudovernik X. (1989). - « Amélioration du comportement dynamique des Aménagements Hydroélectriques par utilisation des paramètres de pression ou de niveau d'eau dans la boucle de régulation ». OZE, Jg.42 Heft 10.

[12] Irving E., Libaux A. (1998), - « Classical/Advanced controls for Hydraulic Power Plants ». Perspectives in Control, Theory and Applications, Springer, CNRS Paris.

[13] Nicolas J., Libaux A., Plandue J.L. (1998), - « Control Systems of Hydro Plants : from the design of high performance systems to their identification for power system study models », Modelling Testing and Monitoring III, Aix-en-Provence. 